\title{
Multi-Agent Based Operation and Control of Isolated Power System with Dispersed Power Sources including New Energy Storage Device
}

\author{
T. Hiyama ${ }^{1}$, T. Nagata ${ }^{2}$ and T. Funabashi ${ }^{3}$ \\ ${ }^{1}$ Department of Electrical \& Computer Engineering \\ Kumamoto University \\ Kumamoto, 860-8555 (Japan) \\ phone:+81 96342 3614, fax:+81 96342 3630, e-mail: hiyama@eecs.kumamoto-u.ac.jp \\ ${ }^{2}$ Hiroshima Institute of Technology \\ Hiroshima, 731-5193 (Japan) \\ e-mail: nagata@cc.it-hiroshima.ac.jp \\ ${ }^{3}$ Meidensha Corporation \\ Tokyo, 103-8515 (Japan) \\ e-mail: funabashi-t@honsha.meidensha.co.jp
}

\begin{abstract}
This paper presents a multi-agent based operation and control of isolated power systems with dispersed power sources such as photo-voltaic units, wind generation units, diesel generation units, and new energy storage devices such as the Energy Capacitor System (ECS). An advanced automatic generation control (AGC) scheme has been proposed through the coordination between the diesel unit and the ECS with small capacity. All the required information for the proposed multiagent based AGC is distributed among intelligent agents through the computer network. All the AGC function is achieved by using the proposed multi-agent based control system. To demonstrate the efficiency of the proposed multiagent based scheme, analog simulator tests have been performed. Simulations results indicate that the AGC performance has been highly improved by the proposed multiagent based AGC scheme even in the existence of the communication delay time through computer networks.
\end{abstract}

\section{Key words}

Multi-agent system, electrical double layer capacitor, Energy Capacitor System, automatic generation control (AGC), dispersed power source.

\section{Introduction}

A number of new distributed power generation technologies, such as the photo-voltaic generation, the wind turbine generation, the micro gas turbine generation, the fuel cells, and the energy storage devices, are currently available to offer integrated performance and flexibility for the power system operation [1-4].

This paper presents a multi-agent based operation and control for isolated power systems with dispersed power sources such as photo-voltaic(PV) units, wind generation units, diesel generation units, and a new energy storage device such as the Energy Capacitor System (ECS). The Energy Capacitor System (ECS) consists of electrical double-layer capacitors [5-7]. The power generation from the photo-voltaic units and also from the wind generation units depends on the environmental factors such as the insolation and the wind velocity, therefore, the complete regulation of the power from these units is quite difficult. To overcome this situation, a new energy storage device, the Energy Capacitor System (ECS) is coordinated with the diesel units for the proposed multiagent based automatic generation control $[8,9]$.

The fast charging/discharging operation is available on the Energy Capacitor System. Therefore, the variations of power from the wind turbine units and also from the PV units can be absorbed through the charging or the discharging operation of the ECS. In addition, the variation of power consumption at the variable load can also be absorbed through the charging/discharging operation of the ECS. A small sized ECS is considered in this study, therefore, the continuous charging or discharging operation is not available on the ECS because of its restricted capacity. Therefore, the power regulation on the diesel units is inevitable to keep the stored energy of the ECS in a proper range for continuing the automatic generation control (AGC) on the ECS. In the proposed AGC scheme, the ECS provides the main function of AGC scheme and the diesel units provide a supplementary function of the AGC scheme. Namely, a coordinated AGC has been proposed between the ECS and the diesel units for balancing the total power generation and the total power demand in the isolated power systems. The proposed multi-agent system consists of three types of intelligent agents: monitoring agents for the distribution of required information through the computer network, the control agents for the charging/discharging operation on the ECS and also for the power regulation on the diesel units, and finally a supervisor agent for the coordination between the ECS and the diesel units.

Real time simulations have been performed on the Analog Power Simulator at the Research Laboratory of 
the Kyushu Electric Power Co. to demonstrate the efficiency of the proposed multi-agent based AGC scheme. Personal computer (PC) based real time modules such as the ECS module, the photo-voltaic module, the wind turbine generator module have been also developed in the Matlab/Simulink environment for the parallel simulations with the analog simulator [10]. All the PC based modules have DSP boards with AD/DA conversion units for the interfaces to and from the analog simulator.

\section{Configuration of Study System}

For the study system, a $6.6 \mathrm{kV}$ distribution system is considered as shown in Fig. 1 [8]. Several dispersed power sources, such as the photo-voltaic unit, the wind turbine unit, the diesel unit, and the ECS for the energy storage, are installed in the target area. After starting the real time simulations on the analog simulator with the PC based modules, the circuit breaker (CB) is opened to shift the target system to an isolated power system.

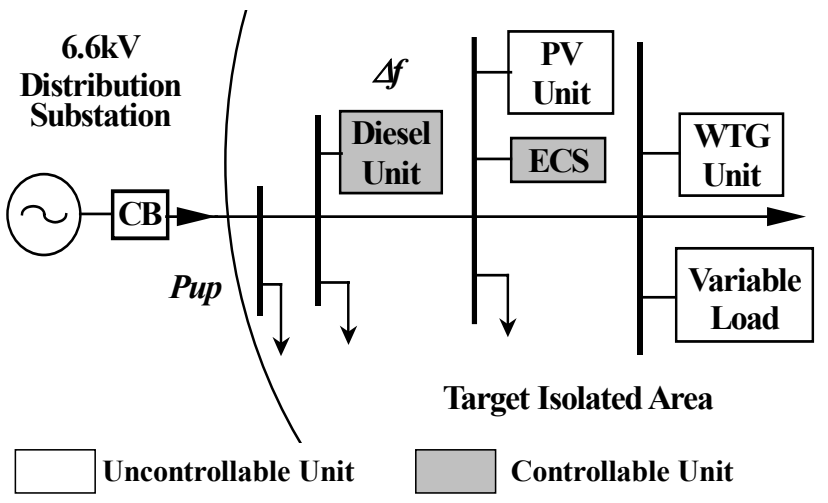

Fig. 1. 6.6kV Distribution System

In the first place, the target system is connected to the upper system. Therefore, the load following operation is taken into account, where the power supply Pup from the upper system is regulated to a pre-specified level by the regulated generation on the diesel unit and also by the charging/discharging operation on the ECS under typical variations of power demand in the target area and also against the power variation from the photo-voltaic (PV) unit and also from the wind turbine generation (WTG) unit. In this study, the capacity of each unit is set as follows: $200 \mathrm{~kW}$ for the PV unit, $1000 \mathrm{~kW}$ for the WTG unit, $5000 \mathrm{~kW}$ for the diesel unit, and $5 \mathrm{kWh}$ for ECS. The maximum charging/discharging power of the ECS is set to $1000 \mathrm{~kW}$.

After opening the circuit breaker (CB), the study system is shifted to an isolated power system. Therefore, the regulation should be shifted to the automatic generation control (AGC) from the former load following operation. For balancing the total power generation and the total power demand, the output power from the ECS and also the output power from the diesel unit should be regulated.

The system frequency $\Delta f$ is monitored on the diesel unit for the proposed agent based AGC. For the realization of the proposed regulations, computer networks are required for the distribution of the required information. Namely, the proposed regulations have been achieved based on the multi-agent system set on the computer networks.

\section{Multi-Agent Based Operation}

Before the isolation of the study system from the upper system, the power supply Pup from upper system is regulated by the charging/discharging operation on the ECS. The diesel unit is also utilized for the coordination with the ECS. A multi-agent based control system has been proposed on the computer networks for the load following operation [9]. The placement of intelligent agents is illustrated in Fig. 2.

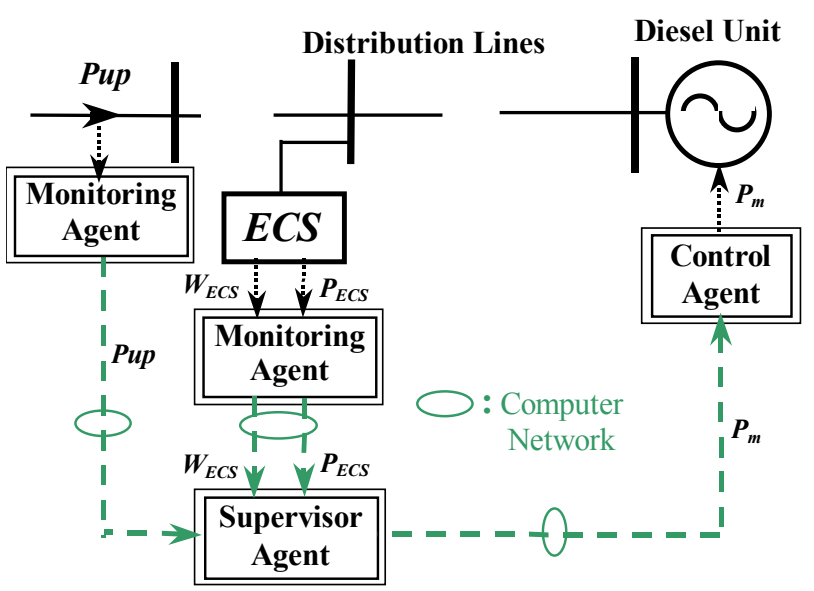

Fig. 2. Intelligent Agents for Load Following Operation

After isolating the study system from the upper system, the purpose of the regulation should be shifted from the load following operation to the automatic generation control (AGC). In the automatic generation control, (AGC) the system frequency monitored on a diesel unit is regulated to its rated frequency by balancing the total generation and the total demand in the isolated power system. In this paper, the main function of the AGC is achieved through the charging/discharging operation on the ECS with the coordination from the power regulation on the diesel units $[10,11]$. Fig. 3 illustrates the configuration of the multi-agent based AGC system for the isolated power systems with dispersed power sources.

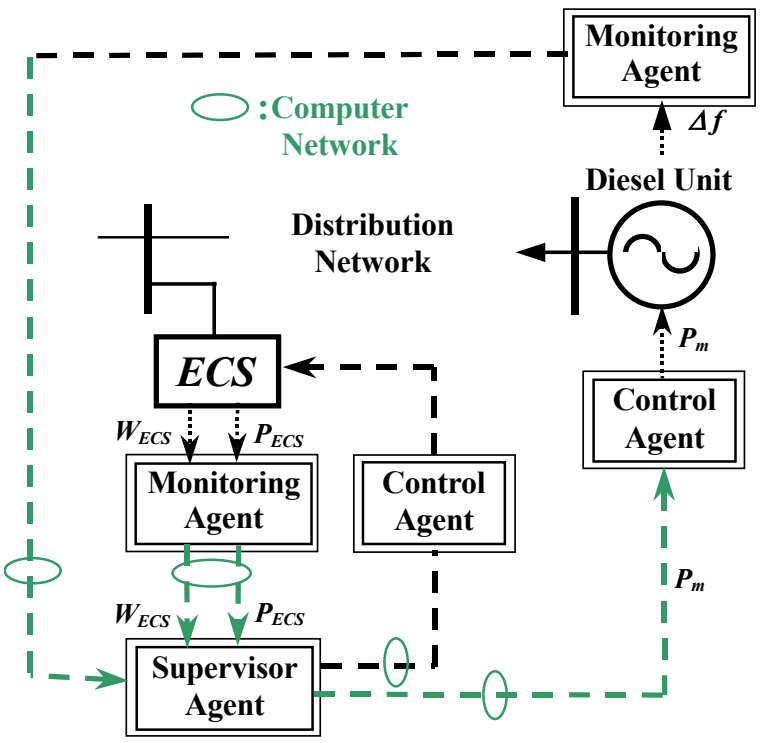

Fig. 3. Intelligent Agents for AGC 


\section{A. Conventional AGC on Diesel Unit}

In the conventional scheme, the AGC is performed on the diesel units to regulate the power generation following the monitored frequency deviation $\Delta f$ on themselves. The regulation is a decentralized one using only the local information. Fig. 4 illustrates the configuration of the AGC system based on the Flat Frequency Control(FFC) with a PI control loop.

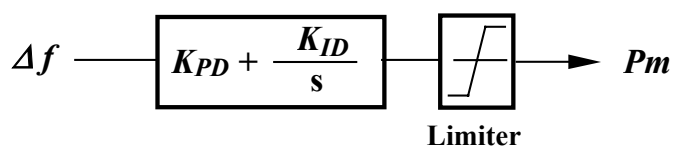

$\Delta f:$ Frequency Deviation of Diesel Unit

$\boldsymbol{P}_{\boldsymbol{m}}$ : Output Setting of Diesel Unit

Fig. 4. Configuration of AGC on Diesel Unit

\section{B. Coordinated AGC between ECS and Diesel Unit}

In this study, a new AGC scheme has been proposed considering the coordination between the ECS and the diesel units. The basic configuration of the proposed AGC controller for the ECS on the supervisor agent is shown in Fig. 5. The configuration is the same as that in Fig. 4, where the diesel units are utilized for the AGC. The charging/discharging level $P S_{E C S}$ is specified by using the frequency deviation $\Delta f$ monitored on one of the diesel units. The monitored frequency deviation $\Delta f$ is transferred to the supervisor agent to determine the charging/discharging level on the ECS. Here, it must be noted that there always exists the communication delay time for the distribution of the required information on the computer network. Following the control signal $P S_{E C S}$ from the $P I$ control loop, the charging/discharging operation is performed on the ECS for the frequency regulation.

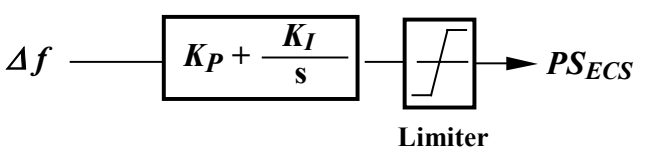

$\Delta f:$ Frequency Deviation of Diesel Unit $\boldsymbol{P S}_{\boldsymbol{E}} \boldsymbol{C S}$ : Output Setting of ECS

Fig. 5. AGC Controller for ECS on Supervisor Agent

Because of the specific feature of the ECS dynamics, the fast charging/discharging operation is available on the ECS. Therefore, the variations of power generation from the wind turbine units and also from the PV units, in addition, the variation of demand power on the variable loads can be efficiently absorbed through the charging or the discharging operation on the ECS. A small sized ECS is considered in this study. Therefore, the regulation of the power generation from the diesel units is required to keep the stored energy level of the ECS in a proper range. Fig. 6 illustrates the configuration of the coordinated controller for the diesel unit on the supervisor agent. In the proposed AGC scheme, the ECS provides the main function of AGC and the diesel units provide a supplementary function to support the charging/discharging operation on the ECS. The coordinated AGC between the ECS and the diesel units has been considered to balance the power demand and the total power generation. Namely, the power generation from the diesel units is regulated to maintain the stored energy level on the ECS for the uninterruptible AGC on the small sized ECS. The power regulation command $\mathrm{Pm}$ is transferred to the control agent on the diesel units though the computer network for the regulation of the power from the diesel units. Here, it must be noted that there exists the communication delay time for transferring the information through the computer network.

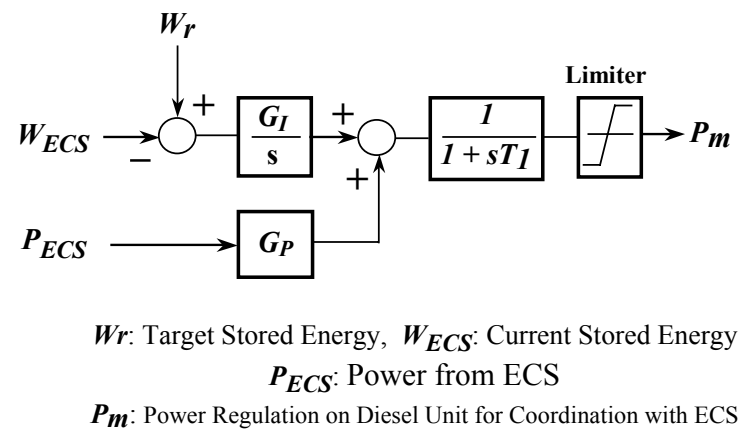

Fig. 6. Coordinated AGC Controller for Diesel Unit on Supervisor Agent

For the realization of the proposed coordinated AGC scheme, a multi-agent system has been utilized as shown in Fig. 3. The required AGC performance is achieved through the charging/discharging operation on the ECS following the frequency deviation $\Delta f$ monitored on one of the diesel units. As shown in Fig. 3, three different types of intelligent agents are defined in the proposed multiagent based AGC system: monitoring agents for the distribution of required information through the computer network, the control agents for the charging/discharging operation on the ECS and also for the power regulation on the diesel units, and finally the supervisor agent for the coordination between the ECS and the diesel units. These three agents can communicate each other through the computer network to achieve the better AGC performance.

\section{Setting of PC Based Agents}

To demonstrate the efficiency of the proposed multiagent based AGC, analog simulator tests have been performed at the Research Laboratory of the Kyushu Electric Power Co. The setting of the PC based intelligent agents is illustrated in Fig. 7. Three personal computers have been utilized for the real time simulations in parallel with the analog simulator. All the personal computers have been linked through the computer network to share the information required for the AGC of the isolated system. In addition, physical interfaces, i. e., $\mathrm{AD} / \mathrm{DA}$ conversion interfaces, have been also required for connecting the personal computer based modules together with the corresponding agents to the analog simulator. For distributing the required information among the intelligent agents through the computer network, the file sharing systems have been also developed in the Matlab/Simulink environment.

Fig. 8 shows the overview of the Analog Power Simulator at the Research Laboratory of the Kyushu 
Electric Power Co. together with the PC based modules and agents.
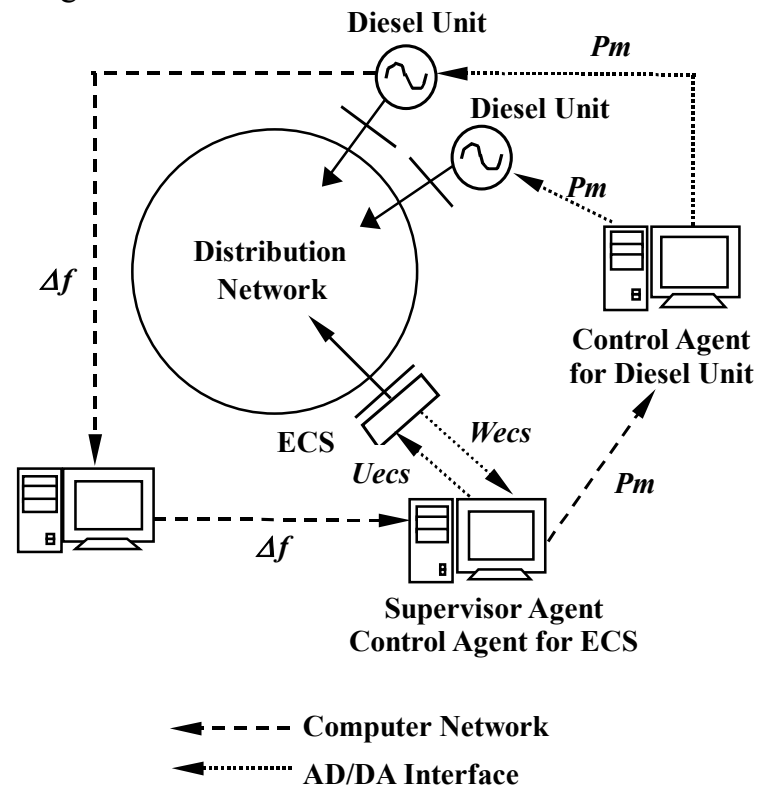

Fig. 7. Setting of PC Based Agents for Analog Simulator Tests

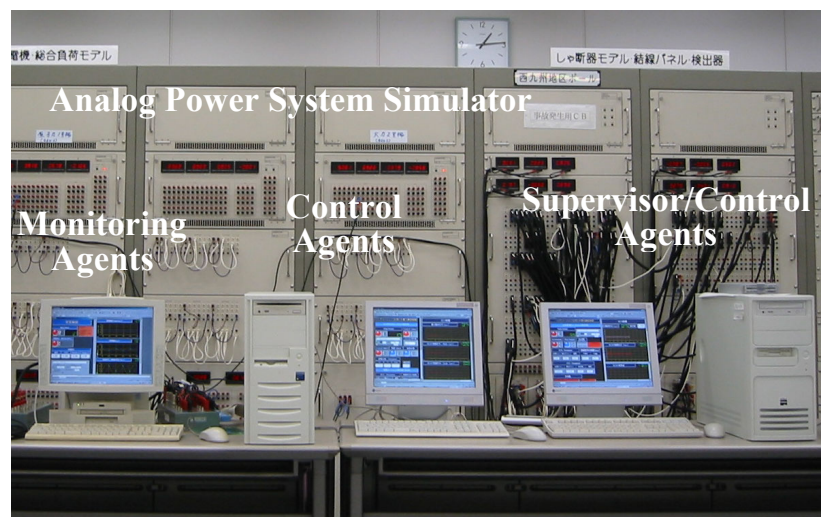

Fig. 8. Overview of Analog Power System Simulator with PC based Intelligent Agents

\section{Analog Simulator Tests}

Real time simulations have been performed to demonstrate the efficiency of the proposed multi-agent based AGC and also to investigate the effect of the communication delay on the AGC performance. In addition, a hierarchical controller has been proposed to overcome the situation of communication deficiency.

\section{A. Typical Simulation Results}

Fig. 9 to Fig. 13 illustrates the typical simulation results for several AGC schemes including the proposed one. The frequency deviation $\Delta f$ measured on the diesel unit is regulated by balancing the total power generation and the total power demand in the study isolated system. In the figures, the frequency deviation $d f$, the power $P_{D G}$ from the diesel unit, the power $P W T$ from the wind turbine generating unit, the pitch angle beta of the wind turbine blade, the power $P P V$ from the photo-voltaic unit, the power PECS from the ECS, the stored energy $W_{E C S}$ on the ECS, the power PLOAD on the variable load, are illustrated from the top to the bottom.
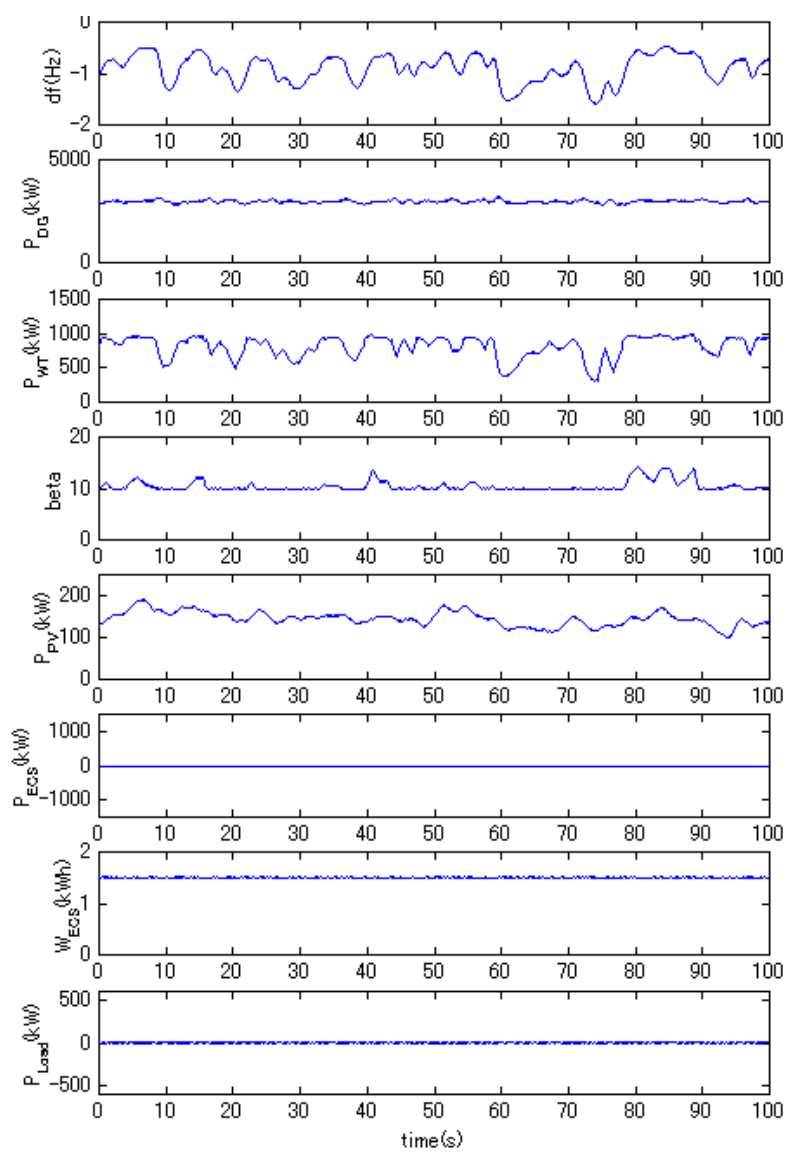

Fig. 9. System Responses without AGC
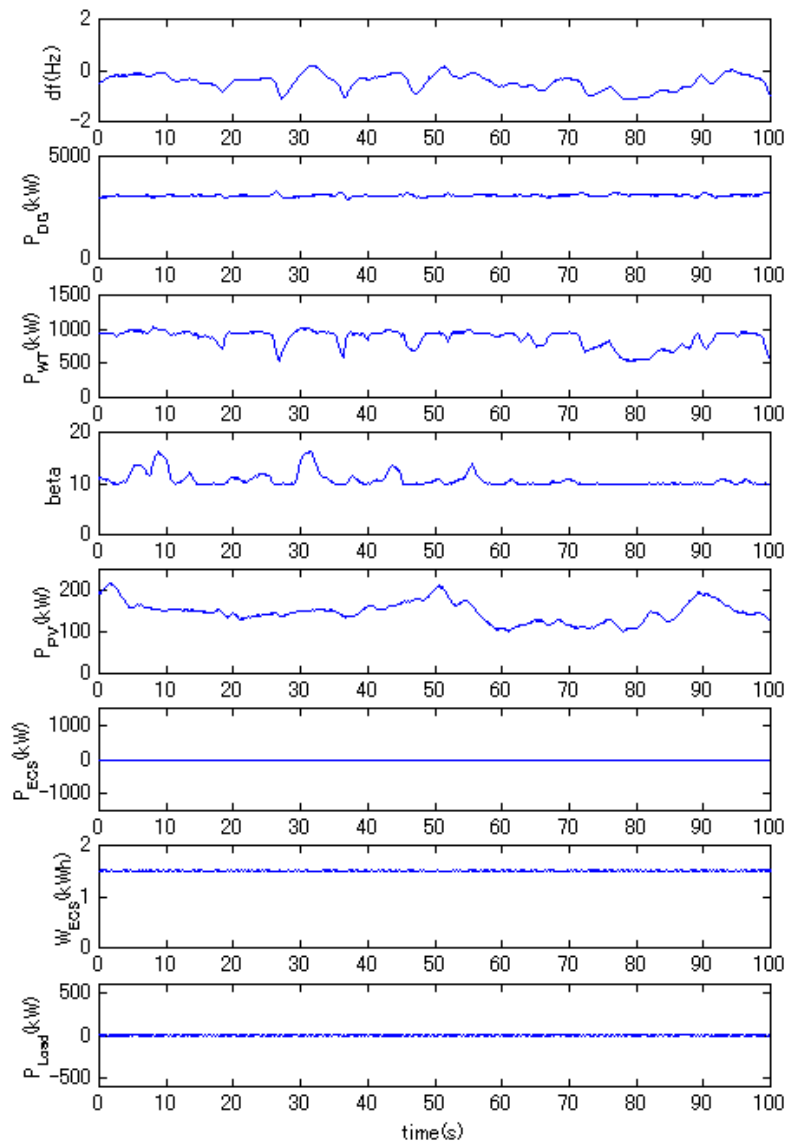

Fig. 10. Conventional AGC Performance on Diesel Unit 

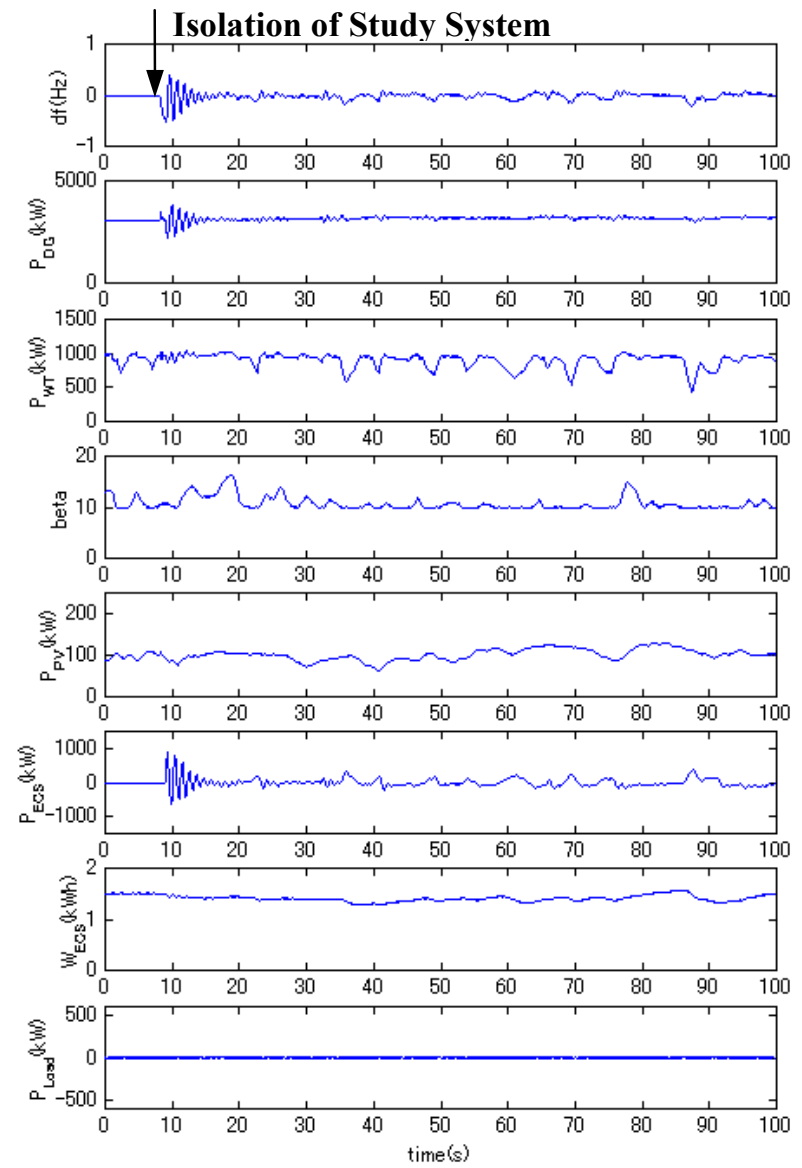

Fig. 11. Proposed Multi-Agent Based AGC Performance just after Shfting to Isolated System
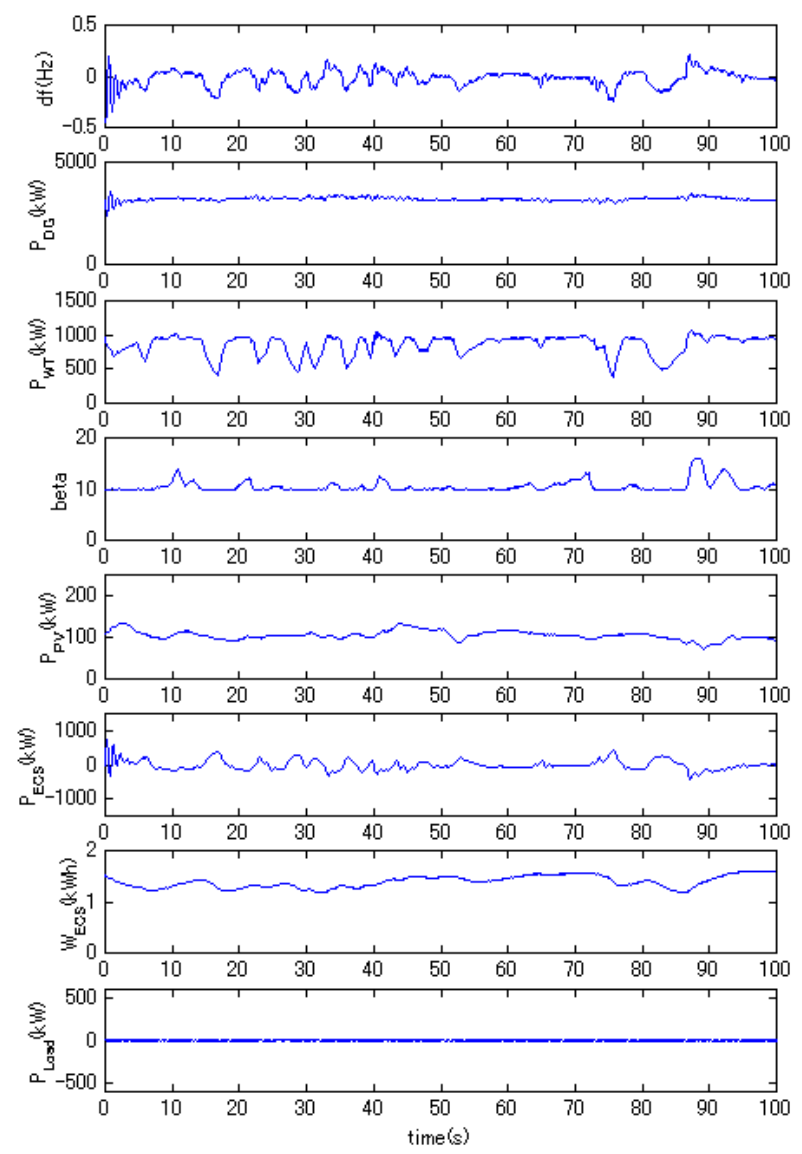

Fig. 12. Proposed Multi-Agent Based AGC Performance

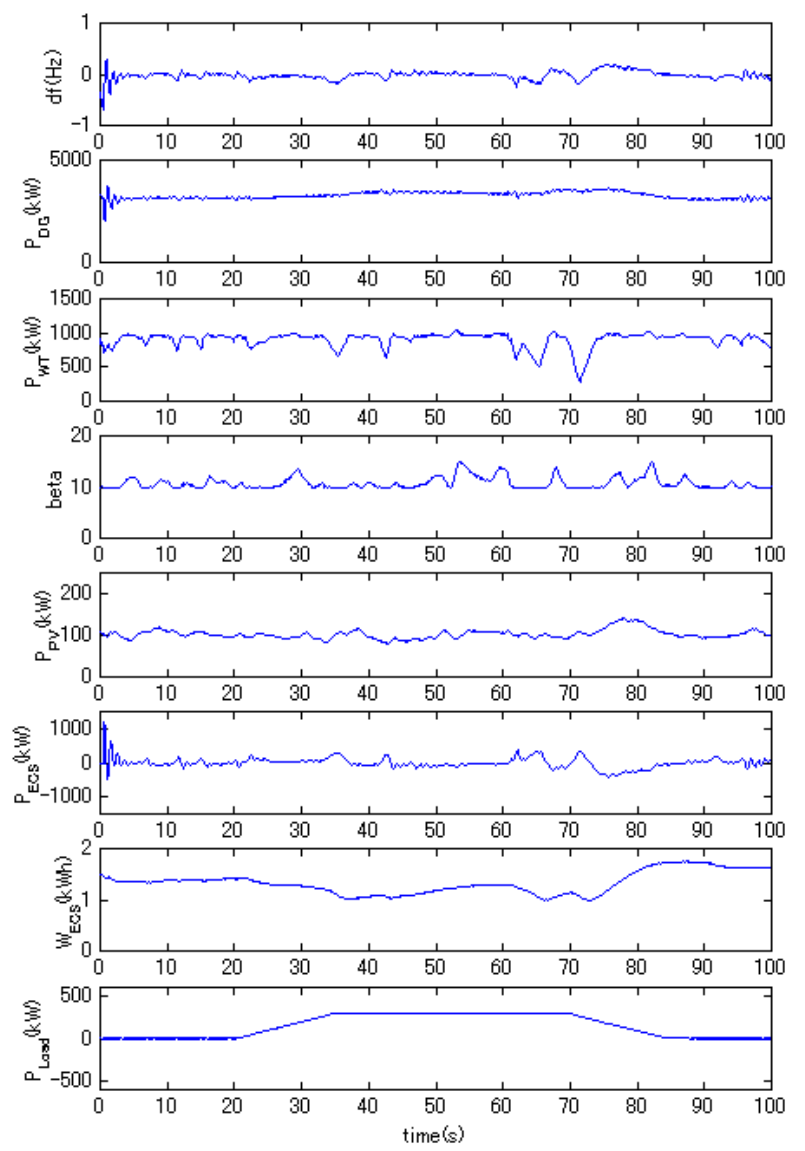

Fig. 13. Proposed Multi-Agent Based AGC Performance for Ramp Load Change

Without the AGC, the system frequency is going down by around $1 \mathrm{~Hz}$ after the isolation of the study system from the upper system as shown in Fig. 9 because of the exceeded power demand from the power generation.

In the case of the conventional AGC on the diesel unit, the frequency deviation is relatively high as shown in Fig. 10. However, when applying the proposed multi-agent based AGC, the frequency deviation is highly reduced as shown in Fig. 11 and Fig. 12. The relatively rapid variations of the power outputs from the wind turbine generating unit and the photo-voltaic unit have been absorbed by the charging/discharging operation on the ECS. Therefore, the frequency variation is kept within a small range as shown in these figures. The shift of the control from the load following control to the AGC has been done without any problem as shown in Fig. 11. Fig. 12 shows the responses for a ramp load change. The stored energy level $W_{E C S}$ has been kept to the prespecified level for the ramp load change by the coordinated regulation of the power from the diesel unit.

The estimated communication delay time is around 40 to $50 \mathrm{~ms}$ for the proposed multi-agent $\mathrm{AGC}$ system on the computer network during the analog simulator tests. There exists no significant degradation of control performance by the existing communication delay. However, for the longer communication delay, the control performance should be degraded or deteriorated not to be able to perform the proper AGC. In such situations, the compensation must be required to keep the proper AGC performance. 
The following indices have been proposed to evaluate the AGC performance:

$$
\begin{aligned}
& \text { Javerage }=\frac{\Delta \text { faverage }}{\text { Average of } P_{D E V}} \times 10^{3} \\
& J \max =\frac{\Delta f \max }{\text { Maximum of } P_{D E V}} \times 10^{3}
\end{aligned}
$$

where

$$
\begin{aligned}
& P_{D E V}=\left|P_{S U M}-P_{S U M} *\right| \\
& P_{S U M}=P_{W T}+P_{P V}+P_{L O A D}
\end{aligned}
$$

Here, it must be noted that $P_{S U M} *$ is the average of the PSUM. By using the indices Javerage and Jmax, the AGC performance can be evaluated properly even under the different situations with respect to the size of the variations of the power outputs from the photovoltaic and the wind turbine units and the variable load power consumption.

Table 1 indicates the values of the performance indices related to the simulation results shown from Fig. 9 to Fig. 13. The AGC performance is highly improved by applying the proposed multi-agent based AGC scheme.

Table 1. Evaluation of AGC Performance

\begin{tabular}{c|c|c|c}
\hline $\begin{array}{c}\text { Type of } \\
\text { Control }\end{array}$ & $\begin{array}{c}\text { Variable } \\
\text { Load }\end{array}$ & $\begin{array}{c}\Delta \text { faverage } \\
(\text { Javerage })\end{array}$ & $\begin{array}{c}\Delta \text { fmax } \\
(\text { Jmax })\end{array}$ \\
\hline none & none & 0.908 & 1.600 \\
& & $(1.156)$ & $(1.616)$ \\
\hline Conventional & none & 0.321 & 1.056 \\
& & $(0.329)$ & $(0.968)$ \\
\hline Proposed & none & 0.058 & 0.256 \\
& & $(0.060)$ & $(0.205)$ \\
\hline Proposed & ramp & 0.052 & 0.238 \\
& & $(0.052)$ & $(0.187)$ \\
\hline Proposed & random & 0.077 & 0.575 \\
& & $(0.093)$ & $(0.506)$ \\
\hline
\end{tabular}

\section{B. Effect of Communication Delay Time on AGC}

To clarify the effect of the communication delay, additional delay time is considered for the existing delay time around 40 to $50 \mathrm{~ms}$. Fig. 14 gives the system responses after adding the delay of $30 \mathrm{~ms}$. The total communication delay might be around 70 to $80 \mathrm{~ms}$ in this case. The AGC performance has been deteriorated and the isolated system becomes unstable as shown in Fig. 14. When considering the actual installation of the proposed multi-agent based AGC scheme, the stable operation of the proposed AGC scheme might be required for the communication delay up to $0.1 \mathrm{~s}$. To improve the AGC performance, the compensation of the delay should be required.

\section{Compensation of Communication Delay Time}

The communication delay can be expressed by the exponential function $e^{-s T d e l a y}$ where $T_{\text {delay }}$ gives the communication delay time. Therefore, the compensator should be approximately defined as follows:

$$
e^{s T_{\text {delay }}} \cong 1+s T_{\text {delay }} \cong 1+\frac{s T_{\text {delay }}}{1+s T}
$$
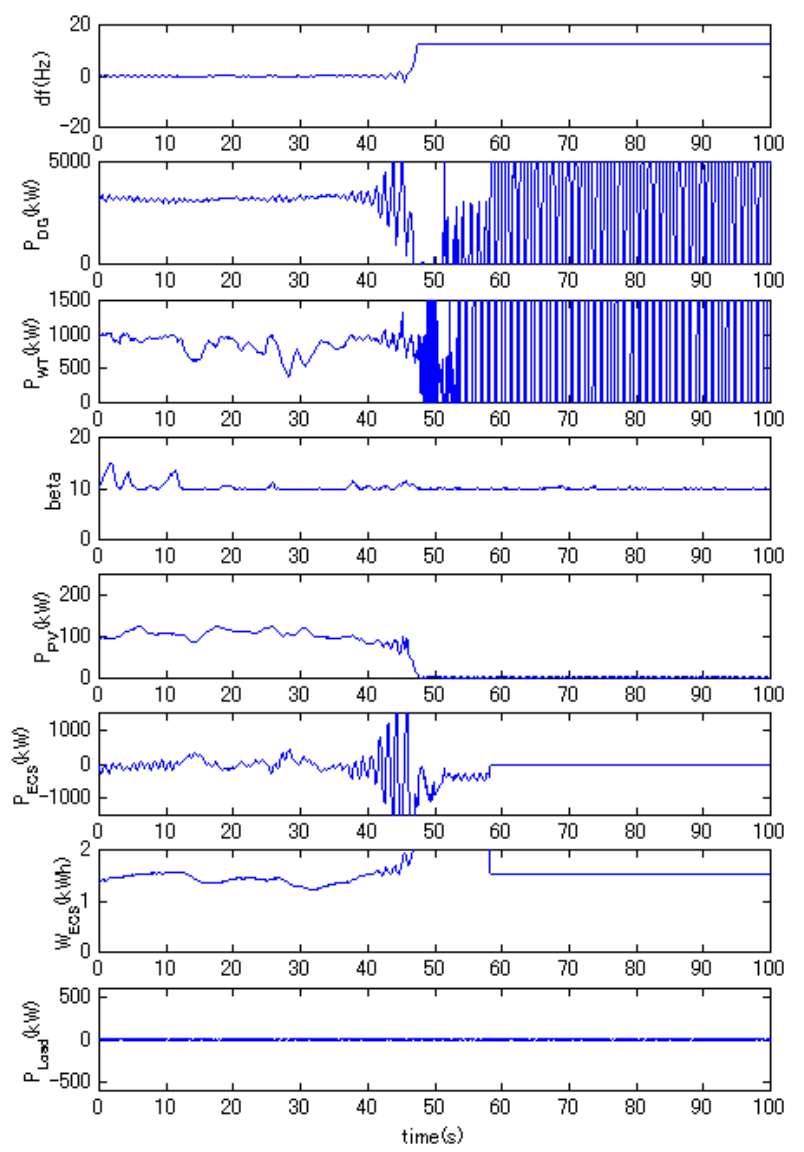

Fig. 14. System Collapse under Longer Communication Delay

As shown in the final approximation of the compensator function, the quasi-differential operator is utilized considering the existence of the higher frequency noise components included in the monitored frequency.

Table 2 indicates the efficiency of the proposed compensation for the additional communication delay time. As shown in the Table, the reliable opretion of the proposed AGC schems is possible under the existence of the additional delay time up to $0.1 \mathrm{~s}$.

Fig. 15 shows the system responses under the existence of the additional communication delay time of $0.09 \mathrm{~s}$, where the compensator is added to the proposed multi-agent system. In this case, the total delay time might be 0.14 to $0.15 \mathrm{~s}$. The feasibility of the proposed multi-agent based AGC scheme should be highly increased by adding the compensator to the proposed AGC system.

Table 2. Performance of Proposed AGC with Compensator

\begin{tabular}{c|c|c|c}
\hline $\begin{array}{c}\text { Additional } \\
\text { Delay Time } \\
(\mathrm{s})\end{array}$ & $\begin{array}{c}\text { Variable } \\
\text { Load }\end{array}$ & $\begin{array}{c}\Delta \text { faverage } \\
\text { (Javerage) }\end{array}$ & $\begin{array}{c}\Delta \text { fmax } \\
(\text { Jmax })\end{array}$ \\
\hline 0.03 & none & $\begin{array}{c}0.049 \\
(0.061)\end{array}$ & $\begin{array}{c}0.256 \\
(0.228)\end{array}$ \\
\hline 0.05 & none & 0.066 & 0.319 \\
& & $(0.081)$ & $(0.275)$ \\
\hline 0.07 & none & 0.079 & 0.406 \\
& & $(0.096)$ & $(0.352)$ \\
\hline 0.09 & none & 0.073 & 0.263 \\
& & $(0.090)$ & $(0.231)$ \\
\hline
\end{tabular}



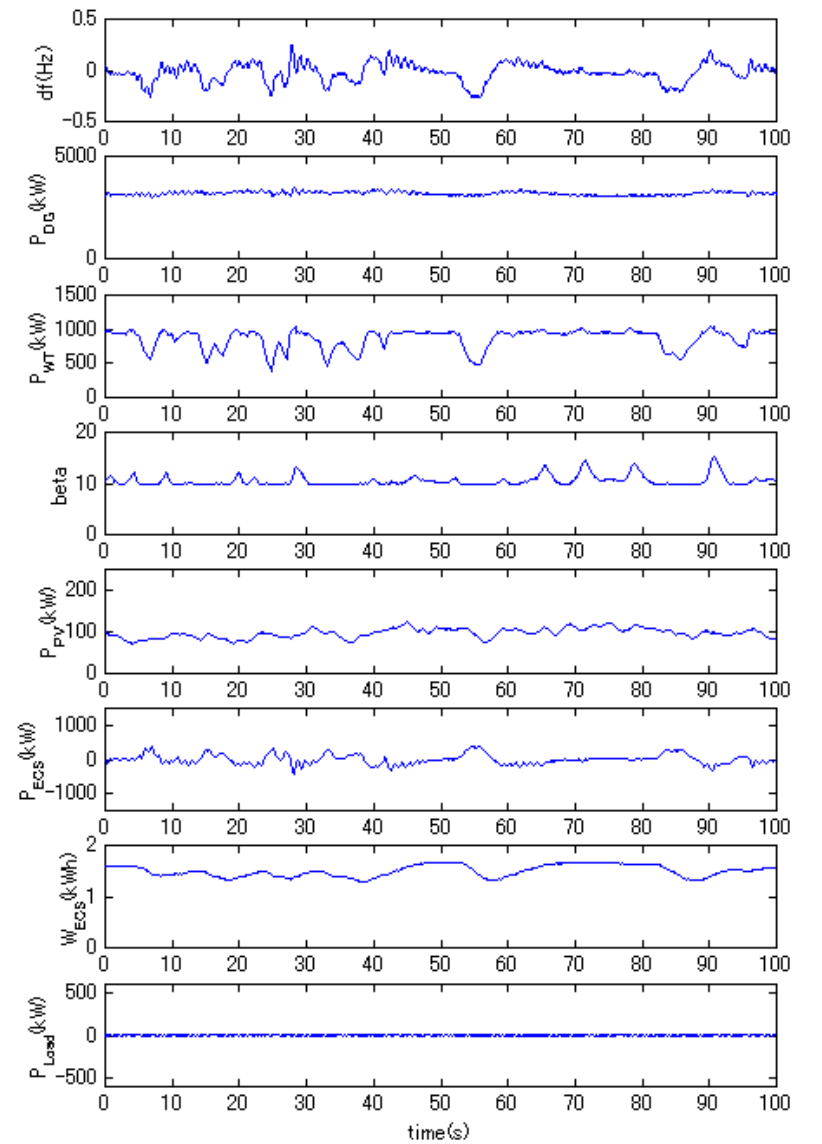

Fig. 15. Effect of Compensation of Communication Delay (Additional Delay Time $=90 \mathrm{~ms}$ )

\section{Hierarchical Control}

Whenever communication failures happen, the proposed multi-agent system can not get the required information through the computer network for the .AGC, therefore, the AGC should be terminated.

To overcome this situation, a hierarchcal control system has been proposed. The proposed hierarchcal control system consists of two types of comntroller: the upper controller which is the same as the multi-agent based AGC proposed in this paper, and the lower controller which is a decentralized AGC controller using only locally measured frequency deviation. The frequency deviation is measured from the zero-cross point of one of the three phase instantaneous voltage components, therefore, the accuracy of the frequency monitoring might be degraded because of the existence of the higher frequency noise signals. In the analog simulator tests, the degradation of the AGC performance has been observed, however, the level is not significant because of the utilization of highly acurate frequency sensor.

\section{Conclusion}

A multi-agent based automatic generation control (AGC) has been proposed for the isolated power systems with dispersed power sources such as photovoltaic units, wind turbine generating units, diesel generating units, and energy capacitor systems (ECS) in this study. In the proposed scheme, the ECS provides the main function for the AGC scheme and the diesel unit provides the supplementary function to coordinate with the ECS. The energy storage level of the ECS is kept in the proper range by the coordination of the diesel unit. The AGC performance has been highly improved by the proposed control scheme even in the existence of the communication delay among the intelligent agents.

\section{Acknowledgement}

The research work has been partially supported by the Grant-in-Aid for Scientific Research (2002-2003) from the JSPS as Project Number 14350148. The authors are also grateful for the collaboration of the Research Laboratory of the Kyushu Electric Power Co. for the utilization of the Analog Power System Simulator.

\section{References}

[1] F. Bonnano, A. Consoli, A. Raciti, B. Morgana, and U. Nocera, "Transient Analysis of Integrated Diesel-WindPhotovoltaic Generation Systems", IEEE Trans. on Energy Conversion, Vol. 14, No. 2, pp.232-238, 1990.

[2] R. Ramakumar, L. Abouzahr, K. Krishnan, and K. Ashenayi, "Design Scenarios for Integrated Renewable Energy Systems", IEEE Trans. on Energy Conversion, Vol. 10, No. 4, pp.736-746, 1995.

[3] G. S. Stavrakakis and G. N. Kariniotakis, "A General Simulation Algorithm for the Accurate Assessment of Isolated Diesel-Wind Turbines Systems Interaction Part I: A General Multimachine Power System Model”, IEEE Trans. on Energy Conversion, Vol. 10, No. 3, pp.577-581, 1995.

[4] G. S. Stavrakakis and G. N. Kariniotakis, "A General Simulation Algorithm for the Accurate Assessment of Isolated Diesel-Wind Turbines Systems Interaction Part II: Implementation of the Algorithm and Case Studies with Induction Generators", IEEE Trans. on Energy Conversion, Vol. 10, No. 3, pp.584-590, 1995.

[5] M. Okamura, "A Basic Study on Power Storage Capacitor Systems", Trans. IEE of Japan, Vol. 115-B, No.5, pp.496503, 1995.

[6] M. Ohshima, M. Shimizu, M. Shimizu, M. Yamagishi, and M. Okamura, "Novel Utility-Interactive Electrical Energy Storage System by Electrical Double Layer Capacitors and an Error Tracking Mode PWM Converter", Trans. IEE of Japan, Vol. 118-D, No. 12, pp.1417-1426, 1998.

[7] T. Hiyama, D. Ueno, S. Yamashiro, M. Yamagishi, and M. Shimizu, "Fuzzy Logic Switching Control for Electrical Double-Layer Energy Capacitor System for Stability Enhancement", in Proceedings of the IEEE PES 2000 Summer Meeting, Vol. 4, pp.2002-2007.

[8] T. Hiyama, K. Tomsovic, M. Yoshimoto, and Y. Hori, "Modeling and Simulation of Distributed Power Sources", in Proceedings of the IPEC'2001, Vol. 2, pp.634-638, 2001.

[9]T. Hiyama, D. Zuo, T. Funabashi, "Multi-Agent Based Load Following Operation of Dispersed Power Sources", in Proceedings of International Symposium on AdCONIP'02 (Advanced Control of Industrial Processes), pp. 61-66.

[10]T. Hiyama, H. Esaki, T. Funabashi, "Experimental Studies on Multi-Agent Based AGC for Isolated Power System with Dispersed Power Sources", in Proceedings of the ISAP'2003 (CD-ROM).

[11]T. Hiyama, H. Esaki, K. Mori, T. Funabashi, "Experimental Studies on Multi-Agent Based Automatic Generation Control for Isolated Power System", in Proceedings of the International Power Engineering Conference 2003, Vol. II, pp.585-589. 